\title{
ОБ АДАПТИВНОМ ПРОКСИМАЛЬНОМ МЕТОДЕ ДЛЯ НЕКОТОРОГО КЛАССА ВАРИАЦИОННЫХ НЕРАВЕНСТВ И СМЕЖНЫХ ЗАДАЧ ${ }^{1}$
}

\author{
Ф. С. Стонякин
}

\begin{abstract}
Для задач безусловной оптимизации хорошо известна концепция неточного оракула, предложенная О. Деволдером, Ф.Глинером и Ю.Е. Нестеровым. В настоящей работе введен аналог понятия неточного оракула (модели функции) для абстрактных задач равновесия, вариационных неравенств и седловых задач. Это позволило предложить аналог известного проксимального метода А. С. Немировского для вариационных неравенств с адаптивной настройкой на уровень гладкости для достаточно широкого класса задач. При этом предусмотрена возможность неточного решения вспомогательных задач проектирования на итерациях метода. Показано, что возникающие погрешности не накапливаются в ходе работы метода. Получены оценки скорости сходимости предложенного метода. Обоснована оптимальность метода с точки зрения теории нижних оракульных оценок. Показано, что предложенный метод применим к смешанным вариационным неравенствам и композитным седловым задачам. Приведен пример, демонстрирующий возможность существенного ускорения метода по сравнению с теоретическими оценками за счет адаптивности критерия остановки.
\end{abstract}

Ключевые слова: неточная модель функции, вариационное неравенство, седловая задача, абстрактная задача равновесия, адаптивный критерий остановки.

F.S. Stonyakin. On the adaptive proximal method for a class of variational inequalities and related problems.

For problems of unconstrained optimization, the concept of inexact oracle proposed by O. Devolder, F. Gleener and Yu. E. Nesterov is well known. We introduce an analog of the notion of inexact oracle (model of a function) for abstract equilibrium problems, variational inequalities, and saddle point problems. This allows us to propose an analog of Nemirovskii's known proximal method for variational inequalities with an adaptive adjustment to the level of smoothness for a fairly wide class of problems. It is also possible to inexactly solve auxiliary problems at the iterations of the method. It is shown that the resulting errors do not accumulate during the operation of the method. Estimates of the convergence rate of the method are obtained, and its optimality from the viewpoint of the theory of lower oracle bounds is established. It is shown that the method is applicable to mixed variational inequalities and composite saddle point problems. An example showing the possibility of an essential acceleration of the method as compared to the theoretical estimates due to the adaptivity of the stopping rule is given.

Keywords: inexact model of a function, variational inequality, saddle point problem, abstract equilibrium problem, adaptive stopping rule.

MSC: 90C33, 90C06, 65K15

DOI: $10.21538 / 0134-4889-2019-25-2-185-197$

\section{1. Введение}

Вариационные неравенства (BH) и седловые задачи часто возникают при решении самых разных проблем оптимизации и имеют многочисленные приложения в математической экономике, математическом моделировании транспортных потоков, теории игр и других разделах математики (см., например, [1]). Исследования в области алгоритмических методов решения вариационных неравенств и седловых задач активно продолжаются (см., например, [2-12]).

\footnotetext{
${ }^{1}$ Теоретические исследования, связанные с концепцией модели функции для вариационных неравенств и седловых задач выполнены при поддержке Российского фонда фундаментальных исследований, грант 18-31-20005 мол-а-вед. Замечание 5 (численные эксперименты для одного вариационного неравенства) выполнены при поддержке Российского научного фонда, проект 18-71-00048.
} 
Наиболее известным аналогом градиентного метода для ВН является экстраградиентный метод Г. М. Корпелевич [13], в качестве одного из современных вариантов которого можно выделить проксимальный зеркальный метод А. С. Немировского [8]. В данной статье предложен аналог этого метода на базе ряда возникших в последние годы новых идей в области алгоритмической оптимизации (см. [14-16]), о которых мы скажем несколько слов.

В 2015 г. Ю.Е. Нестеров в работе [14] предложил так называемые универсалъные градиентные методы для задач выпуклой минимизации. Под универсальностью метода понимается возможность адаптивной настройкой метода на уровень гладкости задачи, что может ускорить работу метода (см. [14, Sec. 5]).

В работе [16] предложен универсальный аналог метода А. С. Немировского для вариационных неравенств. Для оператора $g: Q \rightarrow \mathbb{R}^{n}$, заданного на выпуклом компакте $Q \subset \mathbb{R}^{n}$, под сильным вариационным неравенством понимаем неравенство вида

$$
\left\langle g\left(x_{*}\right), x_{*}-x\right\rangle \leqslant 0,
$$

где $g$ удовлетворяет условию Гельдера

$$
\|g(x)-g(y)\|_{*} \leqslant L_{\nu}\|x-y\|^{\nu} \quad \forall x, y \in Q
$$

для произвольного $\nu \in[0 ; 1]$, причем $L_{0}<+\infty$ (другие константы $L_{\nu}(\nu \neq 0)$ могут быть бесконечными).

Отметим, что в (1.1) требуется найти $x_{*} \in Q$ (это $x_{*}$ и называется решением $\mathrm{BH}$ ), для которого

$$
\max _{x \in Q}\left\langle g\left(x_{*}\right), x_{*}-x\right\rangle \leqslant 0 .
$$

Для монотонного оператора поля $g$ можно рассматривать слабые вариационные неравенства

$$
\left\langle g(x), x_{*}-x\right\rangle \leqslant 0 .
$$

Обычно в (1.4) требуется найти $x_{*} \in Q$, для которого (1.4) верно при всех $x \in Q$. Методика, предложенная в [16], позволяет получить приближенное решение задач (1.1)-(1.4) с точностью $\varepsilon$ и с оценкой сложности (достаточного количества итераций для достижения приемлемого качества решения) $O\left((1 / \varepsilon)^{2 /(1+\nu)}\right)$, которая оптимальна при $\nu=0$ и $\nu=1[7 ; 17 ; 18]$. При этом адаптивность метода [16] на практике может приводить к ускорению работы метода по сравнению с этой оценкой (подробнее об этом написано в замечании 5 ниже).

А. В. Гасников в разд. 3 пособия [15] предложил абстрактную концепцию $(\delta, L)$-модели функции, которая является прямым обобщением известного понятия $(\delta, L)$-оракула О. Деволдера - Ф. Глинера - Ю. Е. Нестерова для задач безусловной оптимизации (см. работу [19], которая активно цитируется оптимизационным сообществом).

О п р е д е л е н и е 1 . Пара $\left(f_{\delta}(y), g_{\delta}(y)\right) \in \mathbb{R} \times E^{*}$ есть $(\delta, L)$-оракул для функции $f:$ $Q \rightarrow \mathbb{R}$ в точке $y$, если для любого $x \in Q$ справедливо неравенство

$$
0 \leq f(x)-f_{\delta}(y)-\left\langle g_{\delta}(y), x-y\right\rangle \leq \frac{L}{2}\|x-y\|^{2}+\delta .
$$

Отметим, что здесь $E$ - пространство, содержащее аргументы функции $f$, а $E^{*}-$ пространство, сопряженное (двойственное) к $E$. Концепция $(\delta, L)$-модели функции [19] в точке отличается от предложенного О. Деволдером, Ю.Е. Нестеровым и Ф. Глинером понятия неточного оракула тем, что линейная функция $\left\langle g_{\delta}(y), x-y\right\rangle$ в $(1.5)$ заменяется на некоторую абстрактную выпуклую функцию.

О п р е д е л е н и е 2. Будем говорить, что имеется $(\delta, L)$-модель функции $f(x)$ в точке $y$ и обозначать эту модель $\left(f_{\delta}(y), \psi_{\delta}(x, y)\right)$, если для любого $x \in Q$ справедливо неравенство

$$
0 \leq f(x)-f_{\delta}(y)-\psi_{\delta}(x, y) \leq \frac{L}{2}\|x-y\|^{2}+\delta,
$$




$$
\psi_{\delta}(x, x)=0 \quad \forall x \in Q
$$

$u \psi_{\delta}(x, y)-$ выпуклая функиия по $x$ для $\forall y \in Q$.

Отметим, что концепция $(\delta, L)$-модели функции из определения 2 позволяет обосновать сходимость обычного и быстрого градиентного методов выпуклой минимизации для достаточно широкого класса задач [15].

Настоящая статья посвящена новому развитию упомянутых выше идей. Мы рассматриваем абстрактную постановку задачи о нахождении точек равновесия и обосновываем возможность использования аналога адаптивного (универсального) метода для вариационных неравенств из [16] для такой постановки. На базе этой идеологии вводится аналог концепции $(\delta, L)$-модели функции для седловых задач. Такой подход позволит распространить методику [16] на более широкий класс задач, в частности на смешанные вариационные неравенства $[4 ; 5]$ и композитные седловые задачи (здесь стоит отметить популярную в оптимизационном сообществе работу [6]). Далее будем рассматривать задачу нахождения решения $x_{*} \in Q$ абстрактной задачи равновесия (неравенство Фань Цзы)

$$
\psi\left(x, x_{*}\right) \geqslant 0 \quad \forall x \in Q
$$

для некоторого выпуклого компакта $Q \subset \mathbb{R}^{n}$, а также функционала $\psi: Q \times Q \rightarrow \mathbb{R}$. Если предположить абстрактную монотонность функционала $\psi$

$$
\psi(x, y)+\psi(y, x) \leqslant 0 \quad \forall x, y \in Q
$$

то всякое решение (1.8) будет также и решением двойственной задачи равновесия

$$
\psi\left(x_{*}, x\right) \leqslant 0 \quad \forall x \in Q .
$$

В общем случае сделаем предположение о существовании решения $x_{*}$ задачи (1.8). Приведем пару примеров задания $\psi$, для которых данное условие заведомо выполнено.

П р и м е $\mathrm{p} 1$. Если для некоторого оператора $g: Q \rightarrow \mathbb{R}^{n}$ положить

$$
\psi(x, y)=\langle g(y), x-y\rangle \quad \forall x, y \in Q,
$$

то (1.8) и (1.10) будут равносильны соответственно стандартным сильному и слабому вариационным неравенствам с оператором $g$.

П р и м е $\mathrm{p} 2$. Для некоторого оператора $g: Q \rightarrow \mathbb{R}^{n}$ и выпуклого функционала $h: Q \rightarrow$ $\mathbb{R}^{n}$ рассмотрение

$$
\psi(x, y)=\langle g(y), x-y\rangle+h(y)-h(x)
$$

приводит к смешанному вариационному неравенству [4;5]

$$
\langle g(y), y-x\rangle+h(x)-h(y) \leqslant 0,
$$

которое в случае монотонности оператора $g$ влечет

$$
\langle g(x), y-x\rangle+h(x)-h(y) \leqslant 0 .
$$

Отметим, что известно немало проксимальных методов для задач нахождения точек равновесия (см., в частности, [20;21] и имеющуюся там библиографию). В [21] предложен проксимальный метод для задач равновесия в гильбертовых пространствах. Однако, как правило, в этих работах лишь исследуются условия сходимости предлагаемых методов без какого-либо обоснования оптимальности скорости сходимости, а также критериев остановки рассматриваемых методов, гарантирующих достижение приемлемого качества решения. Мы же в данной 
статье предлагаем для таких задач аналог метода А.С. Немировского [8]. При этом предлагаемый нами адаптивный критерий остановки за конечное число шагов обеспечивает достижение приемлемого качества приближенного решения по аналогии со случаем вариационных неравенств, рассмотренным в [8]. Важно, что наш подход позволяет учитывать погрешность задания $\psi$, а также погрешности решения вспомогательных задач на итерациях метода.

3 а м е ч а н и е 1. Хорошо известно, что множества решений (1.8) и (1.10) совпадают в предположениях $\psi(x, x)=0$ для всякой точки $x \in Q$, абстрактной монотонности (1.9), выпуклости $\psi$ по первой переменной, а также полунепрерывности $\psi$ снизу по первой переменной и слабой полунепрерывности сверху по второй переменной.

Выделим основные результаты (вклад) данной работы.

- Как обобщение известного для задач оптимизации понятия $(\delta, L)$-оракула О. Деволдера $\Phi$. Глинера - Ю. Е. Нестерова предложено понятие $(\delta, L)$-модели для абстрактной задачи нахождения точек равновесия.

- Для задач нахождения точек равновесия предложен аналог известного для вариационных неравенств и седловых задач проксимального метода А. С. Немировского (см. алгоритм). При этом рассмотрено специальное условие гладкости (2.1), а также предложен адаптивный критерий остановки метода. Адаптивность позволяет применять метод для задач с неизвестной константой $L$, а также ускорять достижение желаемой точности решения.

- Получена оценка скорости сходимости представленного алгоритма, указывающая на его оптимальность с точки зрения теории нижних оракульных оценок (теорема 1 и замечание 2). При этом учитываются погрешности задания функционала $\psi$, а также решения вспомогательных задач на итерациях метода (см. (2.4)). Показано, что погрешности обоих типов не накапливаются в ходе работы метода (в оценках скорости сходимости коэффициенты при параметрах, отвечающих этим погрешностям, не зависят от количества итераций).

- Введено понятие $(\delta, L)$-модели для седловых задач (определение 3 ). Получена оценка скорости сходимости алгоритма для седловых задач, допускающих $(\delta, L)$-модель (теорема 2$)$. При этом также показана возможность учета погрешности задания модели седловой задачи, а также погрешности решения вспомогательных задач на итерациях метода.

- Обоснована возможность применения предложенного метода к смешанным вариационным неравенствам (пример 2) и композитным седловым задачам (пример 3).

Вообще говоря, расстояния до искомого решения задачи мы будем оценивать с помощью понятия расхождения Брэгмана, широко используемого в оптимизации. Напомним, что функция расхождения Брэгмана $V(x, y)$ ( $x$ и $y$ лежат в допустимом множестве $Q$ ) широко используется в оптимизации и вводится на базе 1 -сильно выпуклой относительно нормы $\|\cdot\|$ функции $d$, порождающей расстояния и дифференцируемой во всех точках $x \in Q$

$$
V(x, y)=d(x)-d(y)-\langle\nabla d(y), x-y\rangle \quad \forall x, y \in Q,
$$

где $\langle\cdot, \cdot\rangle$ - скалярное произведение в $\mathbb{R}^{n}$. 1-сильная выпуклость $d$ означает, что

$$
d(x) \geqslant d(y)+\langle\nabla d(y), x-y\rangle+\|x-y\|^{2} \quad \forall x, y \in Q .
$$

В зависимости от постановки конкретной задачи возможны различные подходы к определению прокс-структуры задачи и соответствующего расхождения Брэгмана (см., например, [22]). В случае стандартной евклидовой нормы $\|\cdot\|_{2}$ и расстояния в $\mathbb{R}^{n}$ можно считать, что

$$
V(x, y)=d(x, y)=\frac{1}{2}\|x-y\|_{2}^{2} \quad \forall x, y \in Q .
$$

Однако часто возникает необходимость использовать и неевклидовы нормы. В этом случае расхождение Брэгмана нужно вводить по-другому и его свойства уже не столь тривиальны [22]. 


\section{2. Адаптивный проксимальный метод для абстрактных задач равновесия}

Мы предлагаем адаптивный проксимальный метод для задач (1.8) и (1.10) при следующих предположениях для функционала $\psi$ :

(i) функционал $\psi(x, y)$ выпуклый по первой переменной;

(ii) $\psi(x, x)=0 \quad \forall x \in Q$;

(iii) (абстрактная монотонность) неравенство (1.9);

(iv) (обобщенная гладкость) для некоторых $L>0$ и $\delta>0$

$$
\psi(x, y) \leqslant \psi(x, z)+\psi(z, y)+L V(x, z)+L V(z, y)+\delta \quad \forall x, y, z \in Q .
$$

Отметим, что в случае обычного ВН (1.11) и евклидовой нормы условие (2.1) сводится к неравенству

$$
\langle g(z)-g(y), z-x\rangle \leqslant \frac{L}{2}\|z-x\|^{2}+\frac{L}{2}\|z-y\|^{2}+\delta .
$$

При $\delta=0$ неравенство (2.2) легко проверяется, например, для оператора $g(x)=\nabla f(x)$, где $f$ : $Q \rightarrow \mathbb{R}$ - некоторый выпуклый субдифференцируемый функционал и $\nabla f(x)$ - произвольный субградиент $f$ в точке $x$. Заметим, что при $\delta=0$ похожее на (2.1) условие

$$
\psi(x, y) \leqslant \psi(x, z)+\psi(z, y)+a\|y-z\|^{2}+b\|z-x\|^{2} \quad \forall x, y, z \in Q
$$

( $a$ и $b-$ положительные константы) предложено в [23] и использовалось во многих последующих работах (см., например, [21] и имеющуюся там библиографию). Наш подход позволяет работать с неевклидовой прокс-структурой, а также учитывать неточность $\delta$, что важно, в частности, для идеологии универсальных методов [14;16].

На (2.2) основан предложенный ранее проксимальный метод для вариационных неравенств [16]. Естественно возникает идея обобщить этот метод на абстрактные задачи (1.8) и (1.10) в предположении их разрешимости, а также при условиях (i)-(iv). При этом будем учитывать погрешность $\delta$ в $(2.1)$, а также погрешность $\tilde{\delta}$ решения вспомогательных задач на итерациях согласно одному из достаточно известных в алгоритмической оптимизации подходов [22]:

$$
x:=\arg \min _{y \in Q}^{\tilde{\delta}} \varphi(y), \text { если }\langle\nabla \varphi(x), x-y\rangle \leqslant \tilde{\delta} .
$$

По сути, $x$ из (2.4) есть значение аргумента, обеспечивающее $\tilde{\delta}$-приближенное решение задачи минимизации $\varphi$ на множестве $Q$.

Опишем $(N+1)$-ю итерацию рассматриваемого метода $(N=0,1,2, \ldots)$, выбрав начальное приближение

$$
x^{0}=\arg \min _{x \in Q} d(x)
$$

как точку $x^{0}$, минимизирующую значение прокс-функции $d$ на множестве $Q$. Зафиксируем желаемую точность $\varepsilon>0$ искомого решения задачи (1.10), а также некоторую константу $L^{0} \leqslant 2 L$.

Для краткости будем всюду далее обозначать

$$
S_{N}:=\sum_{k=0}^{N-1} \frac{1}{L^{k+1}} .
$$

Теперь переходим к результату, который описывает скорость сходимости рассматриваемого метода. 
А л г о р и т м. Адаптивный метод для абстрактных задач равновесия.

1. $N:=N+1 ; \quad L^{N+1}:=\frac{L^{N}}{2}$.

2. Вычисляем

$y^{N+1}:=\arg \min _{x \in Q}^{\tilde{\delta}}\left\{\psi\left(x, x^{N}\right)+L^{N+1} V\left(x, x^{N}\right)\right\}$,

$x^{N+1}:=\arg \min _{x \in Q}^{\tilde{\delta}}\left\{\psi\left(x, y^{N+1}\right)+L^{N+1} V\left(x, x^{N}\right)\right\}$

до тех пор, пока не будет выполнено

$$
\begin{aligned}
& \psi\left(x^{N+1}, x^{N}\right) \leqslant \psi\left(y^{N+1}, x^{N}\right)+\psi\left(x^{N+1}, y^{N+1}\right) \\
+ & L^{N+1} V\left(y^{N+1}, x^{N}\right)+L^{N+1} V\left(x^{N+1}, y^{N+1}\right)+\delta .
\end{aligned}
$$

3. Если (2.5) не выполнено, то $L^{k+1}:=2 L^{N+1}$ и повторяем п. 2.

4. Иначе переход к п. 1.

5. Критерий остановки метода:

$$
\sum_{k=0}^{N-1} \frac{1}{L^{k+1}} \geqslant \frac{V\left(x_{*}, x^{0}\right)}{\varepsilon} .
$$

Теорема 1. После остановки рассматриваемого метода для всякого $x \in Q$ будет заведомо выполнено неравенство

$$
-\frac{1}{S_{N}} \sum_{k=0}^{N-1} \frac{\psi\left(x, y^{k+1}\right)}{L^{k+1}} \leqslant \frac{V\left(x, x^{0}\right)}{S_{N}}+2 \tilde{\delta}+\delta \leqslant \varepsilon+2 \tilde{\delta}+\delta,
$$

а такэе

$$
\psi(\tilde{y}, x) \leqslant \frac{V\left(x, x^{0}\right)}{S_{N}}+2 \tilde{\delta}+\delta \leqslant \varepsilon+2 \tilde{\delta}+\delta
$$

npu

$$
\tilde{y}:=\frac{1}{S_{N}} \sum_{k=0}^{N-1} \frac{y^{k+1}}{L^{k+1}}
$$

Отметим, что соответствующие погрешностям параметры $\delta$ и $\tilde{\delta}$ входят в $(2.8)$ и (2.9) с постоянными коэффициентами, которые не растут при увеличении количества итераций. Таким образом, можно сделать вывод о том, что эти погрешности не накапливаются на итерациях метода. Переходим к доказательству теоремы 1.

Д о к а з а т е л ь с т в о. После завершения $(N+1)$-й итерации метода $(N=0,1,2 \ldots)$ ввиду (2.4) имеем

$$
\begin{gathered}
\psi\left(y^{N+1}, x^{N}\right) \leqslant \psi\left(x^{N+1}, x^{N}\right)+L^{N+1} V\left(x^{N+1}, x^{N}\right)-L^{N+1} V\left(x^{N+1}, y^{N+1}\right)-L^{N+1} V\left(y^{N+1}, x^{N}\right)+\tilde{\delta}, \\
\psi\left(x^{N+1}, y^{N+1}\right) \leqslant \psi\left(x, y^{N+1}\right)+L^{N+1} V\left(x, x^{N}\right)-L^{N+1} V\left(x, x^{N+1}\right)-L^{N+1} V\left(x^{N+1}, x^{N}\right)+\tilde{\delta} .
\end{gathered}
$$

Далее, в силу (2.5)

$$
\psi\left(x^{N+1}, x^{N}\right) \leqslant \psi\left(y^{N+1}, x^{N}\right)+\psi\left(x^{N+1}, y^{N+1}\right)+L^{N+1} V\left(y^{N+1}, x^{N}\right)+L^{N+1} V\left(x^{N+1}, y^{N+1}\right)+\delta .
$$

Отметим, что предположение (2.1) гарантирует выполнение условия (2.5) при $L^{N+1} \geqslant L$ после нескольких увеличений $L^{N+1}$ в 2 раза. Просуммировав последние три неравенства, получаем

$$
0 \leqslant \psi\left(x, y^{N+1}\right)+L^{N+1} V\left(x, x^{N}\right)-L^{N+1} V\left(x, x^{N+1}\right)+2 \tilde{\delta}+\delta,
$$


откуда

$$
0 \leqslant \frac{\psi\left(x, y^{N+1}\right)}{L^{N+1}}+V\left(x, x^{N}\right)-V\left(x, x^{N+1}\right)+\frac{1}{L^{N+1}}(2 \tilde{\delta}+\delta)
$$

или после суммирования

$$
-\sum_{k=0}^{N-1} \frac{\psi\left(x, y^{k+1}\right)}{L^{k+1}} \leqslant \sum_{k=0}^{N-1}\left(V\left(x, x^{k}\right)-V\left(x, x^{k+1}\right)\right)+(2 \tilde{\delta}+\delta) S_{N},
$$

откуда и следует доказываемое неравенство (2.8). Наконец, ввиду абстрактной монотонности $\psi(1.9)$ для $\tilde{y}$ из $(2.10)$ имеем

$$
\psi(\tilde{y}, x) \leqslant-\frac{1}{S_{N}} \sum_{k=0}^{N-1} \frac{\psi\left(x, y^{k+1}\right)}{L^{k+1}}
$$

что обосновывает неравенство (2.9).

3 а м е ч а н и е 2 . Ввиду $(2.1)$ и выбора $L^{0} \leqslant 2 L$ гарантированно будет верно $L^{k+1} \leqslant$ $2 L \forall k=\overline{0, N-1}$. Поэтому

$$
S_{N} \geqslant \frac{N}{2 L}
$$

и $(2.8),(2.9)$ означают, что для всякого $x \in Q$ будут верны неравенства

$$
\psi(\tilde{y}, x) \leqslant-\frac{1}{S_{N}} \sum_{k=0}^{N-1} \frac{\psi\left(x, y^{k+1}\right)}{L^{k+1}} \leqslant \frac{2 L V\left(x, x^{0}\right)}{N}+2 \tilde{\delta}+\delta \leqslant \varepsilon+2 \tilde{\delta}+\delta
$$

после выполнения не более чем

$$
O\left(\frac{1}{\varepsilon}\right)
$$

итераций предлагаемого метода. При этом нетрудно проверить, что количество решений вспомогательных задач в п. 2 алгоритма на $N$ итерациях метода не превышает $2 N+\log _{2} \frac{L}{L^{0}}$, т. е. стоимость итерации в среднем будет сопоставимой со стоимостью итерации классического экстраградиентного метода, предполагающей решение двух вспомогательных задач на каждой итерации. Отметим, что оценка (2.12) с точностью до числового множителя оптимальна для вариационных неравенств и седловых задач $[7 ; 17 ; 18]$. Предложенный алгоритм применим и для более широкого класса задач равновесия для функционала $\psi$, удовлетворяющего предположениям (i)-(iv) выше, и с точки зрения количества итераций будет оптимальным с точностью до числового множителя.

З а м е ч а н и е 3. Для обычных слабых вариационных неравенств (1.4) неравенство (2.11) можно заменить на

$$
\max _{x \in Q}\langle g(x), \tilde{y}-x\rangle \leqslant \varepsilon+2 \widetilde{\delta}+\delta
$$

Отметим, что именно (2.13) обычно используют как критерий качества решения вариационного неравенства (см., например, [8]).

З а м е ч а н и е 4. Если нет возможности вычислить точное значение функционала $\psi$, но есть возможность найти $\delta$-приближение $\psi_{\delta}:\left|\psi_{\delta}(x, y)-\psi(x, y)\right| \leqslant \delta$, то в оценках $(2.8),(2.9)$, (2.11) и (2.13) ничего не изменится, кроме величины коэффициента при $\delta$.

3 а м е ч а н и е 5 . В случае гёльдерова оператора поля $g$ (удовлетворяющего (1.2)) известно неравенство (см. замечание 5.1 из [15], а также работу [16])

$$
\langle g(z)-g(y), z-x\rangle \leqslant \frac{L}{2}\|z-x\|^{2}+\frac{L}{2}\|z-y\|^{2}+\frac{\varepsilon}{2}
$$


для некоторой константы $L$, зависящей от $\varepsilon$. На базе интерполяции (2.14) предложенный алгоритм сводится к универсальному методу для вариационных неравенств [16]. Этот метод предполагает адаптивную настройку на уровень гладкости оператора $g$. Заметим, что полученная нами теорема 1 позволяет обобщить этот подход на смешанные вариационные неравенства (пример 2) с гёльдеровым оператором $g$.

Оказывается, что настройка метода на уровень гладкости оператора может позволить ускорить работу метода. В частности, для негладкой задачи $(\nu=0)$ метод на практике может работать со скоростью $O\left(\frac{1}{\varepsilon}\right)$, оптимальной для задач с липшицевым оператором $(\nu=1)$. Приведем один такой пример, связанный с известной задачей Ферма - Торричелли-Штейнера, но при наличии нескольких негладких функциональных ограничений. В общем случае задачи с функциональными ограничениями с использованием принципа множителей Лагранжа сводятся к седловым задачам и соответствующим вариационным неравенствам.

3 а д а ч а. Для заданного набора $r$ точек $A_{k}=\left(a_{1 k}, a_{2 k}, \ldots, a_{n k}\right) n$-мерного евклидова пространства $\mathbb{R}^{n}$ найти такую точку $X=\left(x_{1}, x_{2}, \ldots, x_{n}\right)$, что значение целевой функции

$$
f(x):=\sum_{k=1}^{r} \sqrt{\left(x_{1}-a_{1 k}\right)^{2}+\left(x_{2}-a_{2 k}\right)^{2}+\ldots+\left(x_{n}-a_{n k}\right)^{2}}
$$

будет минимальным для всех точек, удовлетворяющих системе негладких функциональных ограничений:

$$
\begin{gathered}
\varphi_{1}(x)=\alpha_{11}\left|x_{1}\right|+\alpha_{12}\left|x_{2}\right|+\ldots+\alpha_{1 n}\left|x_{n}\right|-1 \leq 0, \\
\varphi_{2}(x)=\alpha_{21}\left|x_{1}\right|+\alpha_{22}\left|x_{2}\right|+\ldots+\alpha_{2 n}\left|x_{n}\right|-1 \leq 0, \\
\ldots \\
\varphi_{m}(x)=\alpha_{m 1}\left|x_{1}\right|+\alpha_{m 2}\left|x_{2}\right|+\ldots+\alpha_{m n}\left|x_{n}\right|-1 \leq 0 .
\end{gathered}
$$

При этом коэффициенты $\alpha_{11}, \alpha_{12}, \ldots, \alpha_{m n}$ образуют матрицу

$$
\left(\begin{array}{cccc}
\alpha_{11} & \alpha_{13} & \ldots & \alpha_{1 n} \\
\alpha_{21} & \alpha_{23} & \ldots & \alpha_{2 n} \\
\ldots \ldots & \ldots & \ldots & \ldots \\
\alpha_{m 1} & \alpha_{m 3} & \ldots & \alpha_{m n}
\end{array}\right) .
$$

Один из элементов каждой строки этой матрицы содержится в промежутке $(1,10)$, а оставшиеся элементы равны 1 . Положим

$$
L(x, \lambda)=f(x)+\sum_{p=1}^{m} \lambda_{p} \varphi_{p}(x), \quad \vec{\lambda}=\left(\lambda_{1}, \lambda_{2}, \ldots, \lambda_{m}\right)
$$

и рассмотрим вариационное неравенство

$$
\left\langle g\left(x_{*}, \vec{\lambda}_{*}\right),\left(x_{*}, \vec{\lambda}_{*}\right)-(x, \vec{\lambda})\right\rangle \leqslant 0 \quad \forall(x, \vec{\lambda}) \in B \subset \mathbb{R}^{n+m}
$$

где

$$
B=\left\{(x, \vec{\lambda}) \mid \sum_{k=1}^{n} x_{k}^{2}+\sum_{p=1}^{m} \lambda_{p}^{2} \leqslant 1\right\}, \quad g(x, \vec{\lambda})=\left(\begin{array}{c}
\nabla f(x)+\sum_{p=1}^{m} \lambda_{p} \nabla \varphi_{p}(x), \\
-\varphi_{1}(x),-\varphi_{2}(x), \ldots,-\varphi_{m}(x)
\end{array}\right) .
$$

Нетрудно понять, что целевой функционал и ограничения не дифференцируемы в бесконечном множестве точек, но имеют ограниченные субдифференциалы в смысле выпуклого анализа. Поэтому оператор $g$ удовлетворяет (1.2) при $\nu=0$. 
Скорость работы универсального метода

\begin{tabular}{|c|c|c|c|c|c|c|c|c|}
\hline & \multicolumn{7}{|c|}{$\varepsilon$} \\
\cline { 2 - 9 } & $1 / 2$ & $1 / 4$ & $1 / 6$ & $1 / 8$ & $1 / 10$ & $1 / 12$ & $1 / 14$ & $1 / 16$ \\
\hline$K$ & 820 & 1554 & 2336 & 3062 & 3882 & 4726 & 5518 & 6258 \\
\hline $\begin{array}{c}\text { Время в формате } \\
\text { ММ:СС }\end{array}$ & $01: 45$ & $03: 18$ & $04: 56$ & $06: 24$ & $08: 07$ & $09: 51$ & $11: 39$ & $13: 04$ \\
\hline
\end{tabular}

Рассмотрим случай $n=10$ переменных и $m=100$ ограничений, начальное приближение

$$
x^{0}=\frac{(0.1,0.1, \ldots, 0.1)}{\|(0.1,0.1, \ldots, 0.1)\|} \in \mathbb{R}^{n+m},
$$

норму и расстояние примем стандартными евклидовыми. При этом координаты точек $A_{k}=$ $\left(a_{1 k}, a_{2 k}, \ldots, a_{n k}\right)$ для $k=1,2, \ldots, 5$ выберем как столбцы матрицы

$$
A=\left(\begin{array}{cccccccccc}
5 & 4 & -7 & -2 & -3 & -8 & 5 & 3 & 8 & 4 \\
-7 & -8 & -9 & -8 & -8 & 6 & -4 & -8 & 4 & -3 \\
-4 & -5 & 8 & 9 & -5 & -4 & -9 & -10 & 1 & 9 \\
7 & -8 & 7 & -8 & -5 & 5 & 3 & -8 & -8 & -6 \\
1 & 9 & -10 & -4 & -8 & -5 & -1 & -2 & 1 & 8
\end{array}\right)
$$

В таблице мы приводим зависимость скорости работы (время и количество необходимых итераций) от желаемой точности $\varepsilon$. Все вычисления были произведены с помощью программного обеспечения CPython 3.7 на компьютере с 3-ядерным процессором AMD Athlon II X3 450 с тактовой частотой 803,5 МГц на каждое ядро. ОЗУ компьютера составляло 8 Гб.

Как видим, метод работает со скоростью, близкой к $O\left(\frac{1}{\varepsilon}\right)$, которая лучше оптимальной теоретической оценки $O\left(\frac{1}{\varepsilon^{2}}\right)$ для задач уровня гладкости $\nu=0$.

\section{3. О концепции $(\delta, \mathbf{L})$-модели функции для седловых задач}

Вариационные неравенства возникают, в частности, при решении седловых задач, в которых для выпуклого по $u$ и вогнутого по $v$ функционала $f(u, v): \mathbb{R}^{n_{1}+n_{2}} \rightarrow \mathbb{R}\left(u \in Q_{1} \subset \mathbb{R}^{n_{1}}\right.$ и $\left.v \in Q_{2} \subset \mathbb{R}^{n_{2}}\right)$ требуется найти такую $\left(u_{*}, v_{*}\right)$, что

$$
f\left(u_{*}, v\right) \leqslant f\left(u_{*}, v_{*}\right) \leqslant f\left(u, v_{*}\right)
$$

для произвольных $u \in Q_{1}$ и $v \in Q_{2}$. Мы считаем $Q_{1}$ и $Q_{2}$ выпуклыми компактами в пространствах $\mathbb{R}^{n_{1}}$ и $\mathbb{R}^{n_{2}}$, и поэтому $Q=Q_{1} \times Q_{2} \subset \mathbb{R}^{n_{1}+n_{2}}$ также есть выпуклый компакт. Для всякого $x=(u, v) \in Q$ будем полагать, что

$$
\|x\|=\sqrt{\|u\|_{1}^{2}+\|v\|_{2}^{2}}
$$

где $\|\cdot\|_{1}$ и $\|\cdot\|_{2}$ - нормы в пространствах $\mathbb{R}^{n_{1}}$ и $\mathbb{R}^{n_{2}}$. Условимся обозначать $x=\left(u_{x}, v_{x}\right), y=$ $\left(u_{y}, v_{y}\right) \in Q$.

Хорошо известно, что для достаточно гладкой функции $f$ по $u$ и $v$ задача (3.1) сводится к вариационному неравенству с оператором

$$
g(x)=\left(\begin{array}{c}
f_{u}^{\prime}\left(u_{x}, v_{x}\right) \\
-f_{v}^{\prime}\left(u_{x}, v_{x}\right)
\end{array}\right) .
$$

Предложим некоторую адаптацию концепции $(\delta, L)$-модели для седловых задач. 
О п р е д е л е н и е 3 . Будем говорить, что функция $\psi(x, y)\left(\psi: \mathbb{R}^{n_{1}+n_{2}} \times \mathbb{R}^{n_{1} \times n_{2}} \rightarrow \mathbb{R}\right)$ есть $(\delta, L)$-модель для седловой задачи (3.1), если для функционала $\psi$ выполнены следующие предположения:

(i) функционал $\psi(x, y)$ выпуклый по первой переменной;

(ii) $\psi(x, x)=0 \quad \forall x \in Q$;

(iii) (абстрактная монотонность) неравенство (1.9);

(iv) (обобщенная гладкостъ) для некоторых постоянных $L>0$ и $\delta>0$ верно неравенство

$$
\psi(x, y) \leqslant \psi(x, z)+\psi(z, y)+L V(x, z)+L V(z, y)+\delta \quad \forall x, y, z \in Q
$$

(v) справедливо неравенство

$$
f\left(u_{y}, v_{x}\right)-f\left(u_{x}, v_{y}\right) \leqslant-\psi(x, y) \quad \forall x, y \in Q .
$$

П р и м е р 3. Предложенная концепция модели функции для седловых задач вполне применима, например, для рассмотренных в статье [6] композитных седловых задач вида

$$
f(u, v)=\tilde{f}(u, v)+h(u)-\varphi(v)
$$

для некоторой выпуклой по $u$ и вогнутой по $v$ субдифференцируемой функции $\tilde{f}$, а также для выпуклых функций простой структуры $h$ и $\varphi$ (для этих функций операция проектирования на множество не очень затратна). В таком случае можно положить

$$
\psi(x, y)=\langle\tilde{g}(y), x-y\rangle+h\left(u_{x}\right)+\varphi\left(v_{x}\right)-h\left(u_{y}\right)-\varphi\left(v_{y}\right)
$$

где

$$
\tilde{g}(y)=\left(\begin{array}{c}
\tilde{f}_{u}^{\prime}\left(u_{y}, v_{y}\right) \\
-\tilde{f}_{v}^{\prime}\left(u_{y}, v_{y}\right)
\end{array}\right)
$$

Действительно, из субградиентных неравенств получаем

$$
\tilde{f}\left(u_{y}, v_{y}\right)-\tilde{f}\left(u_{x}, v_{y}\right) \leqslant\left\langle-\tilde{f}_{u}^{\prime}\left(u_{y}, v_{y}\right), u_{x}-u_{y}\right\rangle, \quad \tilde{f}\left(u_{y}, v_{x}\right)-\tilde{f}\left(u_{y}, v_{y}\right) \leqslant\left\langle\tilde{f}_{v}^{\prime}\left(u_{y}, v_{y}\right), v_{x}-v_{y}\right\rangle .
$$

Поэтому имеем $\tilde{f}\left(u_{y}, v_{x}\right)-\tilde{f}\left(u_{x}, v_{y}\right) \leqslant-\langle\tilde{g}(y), x-y\rangle$, откуда

$$
\begin{gathered}
f\left(u_{y}, v_{x}\right)-f\left(u_{x}, v_{y}\right)=\tilde{f}\left(u_{y}, v_{x}\right)+h\left(u_{y}\right)-\varphi\left(v_{x}\right)-\tilde{f}\left(u_{x}, v_{y}\right)-h\left(v_{x}\right)+\varphi\left(v_{y}\right) \\
=\tilde{f}\left(u_{y}, v_{x}\right)-\tilde{f}\left(u_{x}, v_{y}\right)+h\left(u_{y}\right)+\varphi\left(v_{y}\right)-h\left(v_{x}\right)-\varphi\left(v_{x}\right) \\
\leqslant-\langle\tilde{g}(y), x-y\rangle+h\left(u_{y}\right)+\varphi\left(v_{y}\right)-h\left(v_{x}\right)-\varphi\left(v_{x}\right)=-\psi(x, y) .
\end{gathered}
$$

Из теоремы 1 вытекает

Теорема 2. Если для седловой задачи (3.1) существует $(\delta, L)$-модель $\psi(x, y)$, то после остановки алгоритма получаем точку

$$
\tilde{y}=\left(u_{\tilde{y}}, v_{\tilde{y}}\right):=(\tilde{u}, \tilde{v}):=\frac{1}{S_{N}} \sum_{k=0}^{N_{1}} \frac{y_{k+1}}{L^{k+1}},
$$

для которой верна оценка величинь-качества решения седловой задачи

$$
\max _{v \in Q_{2}} f(\tilde{u}, v)-\min _{u \in Q_{1}} f(u, \tilde{v}) \leqslant \varepsilon+2 \tilde{\delta}+\delta .
$$




\section{4. Заключение}

Таким образом, введенная в работе концепция $(\delta, L)$-модели функции для задач равновесного программирования позволила распространить ранее предложенный в [16] универсальный метод для вариационных неравенств на более широкий класс задач. В частности, методика настоящей работы применима к смешанным вариационным неравенствам [4;5], а также к композитным седловым задачам [6]. При этом была учтена возможность неточного задания функционала $\psi$, а также неточность решения вспомогательных задач проектирования на итерациях метода (см. замечание 4). Показано, что при этом сохраняются оптимальные с точки зрения нижних оракульных оценок скорости сходимости метода, а погрешности обоих типов не накапливаются в ходе итераций метода. Приводится пример, иллюстрирующий возможность ускорения работы предложенного метода по сравнению с теоретическими оценками за счет адаптивного выбора шага и адаптивного критерия остановки (см. замечание 5).

Отметим некоторые открытые вопросы. Хорошо известно, что в общем случае к седловым задачам с помощью метода множителей Лагранжа сводятся задачи выпуклой условной оптимизации. Допустим, что целевой функционал или функционал ограничения в задаче условной оптимизации не имеет липшицева градиента, но при этом для него существует $(\delta, L)$-модель в смысле определения 2. В таком случае ожидается, что соответствующая лагранжиева седловая задача будет иметь $(\delta, L)$-модель в смысле определения 3. Представляет интерес исследовать применимость результатов настоящей работы к задачам условной оптимизации с такими функционалами. Также представляется актуальным вопрос о возможности использования похожей методики для каких-либо классов задач невыпуклой оптимизации, а также для вариационных неравенств с немонотонными операторами.

Благодарности. Автор выражает огромную признательность Александру Владимировичу Гасникову, Павлу Евгеньевичу Двуреченскому и Юрию Евгеньевичу Нестерову за полезное обсуждение и пожелания.

\section{СПИСОК ЛИТЕРАТУРЫ}

1. Facchinei F., Pang J.S. Finite-dimensional variational inequality and complementarity problems. Vols 1 and 2. N Y: Springer-Verlag, 2003. Vol. 1, 693 p. doi: 10.1007/b97543; vol. 2, 704 p. doi: $10.1007 /$ b97544.

2. Антипин А. С. Методы решения вариационных неравенств со связанными ограничениями // Журн. вычисл. математики и мат. физики. 2000. Т. 40, № 9. С. 1291-1307.

3. Антипин А. С., Ячимович В., Ячимович М. Динамика и вариационные неравенства // Журн. вычисл. математики и мат. физики. 2017. Т. 57, № 5. С. 783-800. doi: 10.7868/S0044466917050015.

4. Коннов И. В., Салахутдин Р. А. Двухуровневый итеративный метод для нестационарных смешанных вариационных неравенств // Изв. вузов. Математика. 2017. № 10. С. 50-61.

5. Bao T. Q., Khanh P. Q. Some algorithms for solving mixed variational inequalities // Acta Mathem. Vietnamica. 2006. Vol. 31, no. 1. P. 77-98. doi: 10.1016/j.jco.2014.08.003.

6. Chambolle A., Pock T. A first-order primal-dual algorithm for convex problems with applications to imaging // J. Math. Imag. Vis. 2011. Vol. 40, no. 120. doi: 10.1007/s10851-010-0251-1.

7. Guzman C., Nemirovski A. On lower complexity bounds for large-scale smooth convex optimization // J. Complexity. 2015. Vol. 31, no. 1. P. 1-14. doi: 10.1016/j.jco.2014.08.003 .

8. Nemirovski A. Prox-method with rate of convergence $\mathrm{O}(1 / \mathrm{T})$ for variational inequalities with Lipschitz continuous monotone operators and smooth convex-concave saddle point problems // SIAM J. Optim. 2004. Vol. 15, no. 1. P. 229-251. doi: 10.1137/S1052623403425629.

9. Меленьчук Н. В. Методы и алгоритмы для решения задач математического моделирования на основе вариационных неравенств: дис. ... канд. физ.-мат. наук. Омск, 2011. 123 с.

10. Nesterov Yu. Dual extrapolation and its application for solving variational inequalities and related problems // Math. Program. 2007. Ser. B. P. 319-344. doi: 10.1007/s10107-006-0034-z .

11. Nesterov Yu., Scrimali L. Solving strongly monotone variational and quasi-variational inequalities // Discr. Contin. Dynam. Syst. Ser. A. 2011. Vol. 31, no. 4. P. 1383-1396. doi: 10.3934/dcds.2011.31.1383. 
12. Нестеров Ю. Е. Алгоритмическая выпуклая оптимизация: дис. ... д-р физ.-мат. наук. М.: Моск. физ.-техн. ин-т. 2013. 367 с.

13. Корпелевич Г. М. Экстраградиентный метод для отыскания седловых точек и других задач // Экономика и мат. методы. 1976. Т. 12, № 4. С. 747-756.

14. Nesterov Yu. Universal gradient methods for convex optimization problems // Math. Program. 2015. Ser. A. Vol. 152, no. 1-2. P. 381-404. doi: 10.1007/s10107-014-0790-0.

15. Гасников А. В. Современные численные методы оптимизации. Метод универсального градиентного спуска: учеб. пособие. Москва: Изд-во МФТИ, 2018. 160 с.

16. Гасников А. В., Двуреченский П. Е., Стонякин Ф. С., Титов А. А. Адаптивный проксимальный метод для вариационных неравенств // Журн. вычисл. математики и мат. физики. 2019. Т. 59, № 5. C. 889-894. doi: 10.1134/S0965542519050075.

17. Немировский А. С., Юдин Д. Б. Сложность задач и эффективность методов оптимизации // Москва: Наука. 1979. 384 с.

18. Nemirovski A. Information-based complexity of convex programming [e-resource]. Technion, Fall Semester 1994/95 / The Israel institute of technology faculty of industrial engineering \& management. 268 p. URL: https://www2.isye.gatech.edu/ nemirovs/Lect_EMCO.pdf .

19. Devolder O., Glineur F., Nesterov Yu. First-order methods of smooth convex optimization with inexact oracle // Math. Progr. 2014. Vol. 146, no. 1-2. P. 37-75. doi: 10.1007/s10107-013-0677-5.

20. Антипин А. С. Равновесное программирование: проксимальные методы // Журн. вычисл. математики и мат. физики. 1997. Т. 37, № 11. С. 1327-1339.

21. Ведель Я. И., Семенов В. В. Новый двухэтапный проксимальный алгоритм для решения задачи о равновесии // Журн. вычисл. и прикл. математики. 2015. Т. 118, № 1. С. 15-23.

22. Ben-Tal A., Nemirovski A. Lectures on modern convex optimization. Philadelphia: SIAM, 2001. $500 \mathrm{p}$.

23. Mastroeni G. On auxiliary principle for equilibrium problems // Publicatione del Departimento di Mathematica Dell'Universita di Pisa. 2000. Vol. 3. P. 1244-1258.

Поступила 8.02.2019

После доработки 7.05.2019

Принята к публикации 13.05.2019

Стонякин Федор Сергеевич

канд. физ.-мат. наук, доцент

Крымский федеральный университет им. В. И. Вернадского

г. Симферополь

e-mail: fedyor@mail.ru

\section{REFERENCES}

1. Facchinei F., Pang J. S. Finite-dimensional variational inequality and complementarity problems, vols. 1 and 2. N Y, Springer-Verlag, 2003, vol. 1. 693 p. doi: 10.1007/b97543; vol. 2. 704 p. doi: 10.1007/b97544 .

2. Antipin A.S. Solution methods for variational inequalities with coupled constraints. Comput. Math. Math. Phys., 2000, vol. 40, no. 9, pp. 1239-1254.

3. Antipin A.S., Jaćimović V., Jaćimović M. Dynamics and variational inequalities. Comput. Math. Math. Phys., 2017, vol. 57, no. 5, pp. 784-801. doi: 10.1134/S0965542517050013.

4. Konnov I. V., Salahuddin R. A. Two-level iterative method for non-stationary mixed variational inequalities. Russian Math. (Iz. VUZ), 2017, vol. 61, no. 10, pp. 44-53. doi: $10.3103 / \mathrm{S} 1066369 X 17100061$.

5. Bao T. Q., Khanh P. Q. Some algorithms for solving mixed variational inequalities. Acta Math. Vietnamica, 2006, vol. 31, no. 1, pp. 77-98.

6. Chambolle A., Pock T. A first-order primal-dual algorithm for convex problems with applications to imaging. J. Math. Imag. Vis., 2011, vol. 40, no. 1, pp. 120-145. doi: 10.1007/s10851-010-0251-1.

7. Guzman C., Nemirovski A. On lower complexity bounds for large-scale smooth convex optimization. J. Complexity, 2015, vol. 31, no. 1, pp. 1-14. doi: 10.1016/j.jco.2014.08.003. 
8. Nemirovski A. Prox-method with rate of convergence $\mathrm{O}(1 / \mathrm{T})$ for variational inequalities with Lipschitz continuous monotone operators and smooth convex-concave saddle point problems. SIAM J. Optim., 2004, vol. 15, no. 1, pp. 229-251. doi: 10.1137/S1052623403425629.

9. Melenchuk N. V. Metody i algoritmy dlya resheniya zadach matematicheskogo modelirovaniya na osnove variatsionnykh neravenstv [Methods and algorithms for solving problems of mathematical modeling based on variational inequalities]. Candidate Sci. (Phys.-Math.) Dissertation: 05.13.18, Omsk, 2011, 123 p.

10. Nesterov Yu. Dual extrapolation and its application for solving variational inequalities and related problems. Math. Program., Ser. B., 2007, vol. 109, no. 2-3, pp. 319-344. doi: 10.1007/s10107-006-0034-z .

11. Nesterov Yu., Scrimali L. Solving strongly monotone variational and quasi-variational inequalities. Discrete and Continuous Dynamical Systems. Ser. A., 2011, vol. 31, no. 4, pp. 1383-1396. doi: 10.3934/dcds.2011.31.1383 .

12. Nesterov Yu. E. Algoritmicheskaya vypuklaya optimizatsiya [Algorithmic convex optimization]. Doctor Sci. (Phys.-Math.) Dissertation. Moscow: Mosk. Phys.-Tech. Inst. (State University), 2013. 367 p.

13. Korpelevich G. M. Extragradient method for finding saddle points and other problems. Ekonom. Mat. Metody, 1976, vol. 12, no. 4, pp. 747-756 (in Russian).

14. Nesterov $\mathrm{Yu}$. Universal gradient methods for convex optimization problems. Math. Program. Ser. A, 2015, vol. 152, no. 1-2, pp. 381-404. doi: 10.1007/s10107-014-0790-0 .

15. Gasnikov A. V. Sovremennye chislennye metody optimizatsii. Metod universal'nogo gradientnogo spuska [Modern numerical optimization methods. The universal gradient descent method]. Moscow: MIPT Publ., 2018, 160 p.

16. Gasnikov A. V., Dvurechensky P.E., Stonyakin F.S., Titov A. A. Adaptive proximal method for variational inequalities. Zh. Vychisl. Mat. Mat. Fiz., 2019, vol. 59, no. 5, pp. 889 - 894 (in Russian).

17. Nemirovskii A.S., Yudin D. B. Problem complexity and method efficiency in optimization. Chichester etc.: John Wiley \& Sons., 1983, 388 p. ISBN: 9780471103455. Original Russian text published in Nemirovskii A.S., Yudin D.B. Slozhnost' zadach i effektivnost' metodov optimizatsii. Moscow: Nauka Publ., 1979, 384 p.

18. Nemirovski A. Information-based complexity of convex programming [e-resource]. Technion, Fall Semester 1994/95 / The Israel Institute of Technology Faculty of Industrial Engineering \& Management. 268 p. Available at: https://www2.isye.gatech.edu/ nemirovs/Lect_EMCO.pdf.

19. Devolder O., Glineur F., Nesterov Yu. First-order methods of smooth convex optimization with inexact oracle. Math. Progr., 2014, vol. 146, no. 1-2, pp. 37-75. doi: 10.1007/s10107-013-0677-5.

20. Antipin A. S. Equilibrium programming: Proximal methods. Comput. Math. Math. Phys., 1997, vol. 37, no. 11 , pp. $1285-1296$.

21. Vedel'Ya. I., Semenov V. V. New two-stage proximal algorithm for solving the problem of equilibrium. Zh. Vychisl. Mat. Mat. Fiz., 2015, no. 1 (118), pp. 15-23 (in Russian).

22. Ben-Tal A., Nemirovski A. Lectures on modern convex optimization. Philadelphia, SIAM, 2001.500 p.

23. Mastroeni G. On auxiliary principle for equilibrium problems. In: Daniele P., Giannessi F., Maugeri A. (eds) Equilibrium Problems and Variational Models, Ser. Nonconvex Optim. Its Appl., vol. 68, Boston: Springer, 2003, pp. 289-298. doi: 10.1007/978-1-4613-0239-1_15.

Received February 8, 2019

Revised May 7, 2019

Accepted May 13, 2019

Funding Agency: The theoretical research (the concept of a model of a function for variational inequalities and saddle point problems) was supported by the Russian Foundation for Basic Research (project no. 18-31-20005 mol-a-ved). The research of Remark 5 (numerical experiments for one variational inequality) was supported by the Russian Science Foundation (project no. 18-71-00048).

Fedor Sergeevich Stonyakin, Cand. Sci. (Phys.-Math.), V.I. Vernadsky Crimean Federal University, Simferopol, Republic of Crimea, 295007 Russia, e-mail: fedyor@mail.ru .

Cite this article as: F. S. Stonyakin. On the adaptive proximal method for a class of variational inequalities and related problems, Trudy Instituta Matematiki i Mekhaniki URO RAN, 2019, vol. 25, no. 2, pp. 185-197. 